\title{
Development of Heat Transfer Coefficient and Friction Factor Correlations for Serrated Fins in Water Medium using CFD
}

\author{
K V Ramana Murthy ${ }^{1 *}$, C Ranganayakulu ${ }^{1}$ and T P Ashok Babu ${ }^{2}$ \\ 1. General systems Directorate, Aeronautical Development Agency, Bangalore -560 017, India \\ 2.Mechanical Engineering Dept, National Institute of Technology, Surathkal, -575 025, India.
}

\begin{abstract}
The most popularly used fin types in compact heat exchangers are the serrated fins, wavy fins, louvered fins and plain fins. Amongst these fin types the serrated fins assume lot of importance due to its enhanced thermo-hydraulic performance. Thermo-hydraulic design of CHEs (Compact heat exchangers) is strongly dependent upon the predicted/measured dimensionless performance (Colburn $j$ factor and Fanning friction vs. Reynolds number) of heat transfer surfaces. This paper describes the numerical analysis to study the heat transfer coefficient and friction factor of Serrated fins in water medium. CFD (Computational fluid dynamics) methodology has been used to develop the single phase water heat transfer coefficient and friction factor correlations for serrated fins using ANSYS Fluent 14.5. The results are compared with previous air-cooled models and experimental results of water. The water cooled CFD analysis results shows that the Prandtl number has a large effect on the Nusselt number of the serrated fin geometry. Finally, the generalized correlations are developed for serrated fins taking all geometrical parameters into account. This numerical estimation can reduce the number of tests/experiments to a minimum for similar applications.
\end{abstract}

Key words: Compact heat exchanger, serrated fins, CFD (Computational fluid dynamics) analysis, water medium, thermo-hydraulic performance, colburn $j$ factor, friction factor $f$.

\section{Nomenclature}

$\begin{array}{ll}f & \text { Fanning friction factor, dimensionless } \\ \text { FPI } & \text { Fins per inch } \\ \mathrm{h}, \mathrm{H} & \text { Fin height, } \mathrm{mm} \\ j & \text { Colburn factor }\left(\mathrm{StPr}^{2 / 3}\right), \text { dimensionless } \\ l & \text { Offset strip fin length, mm } \\ R e & \text { Reynolds number, dimensionless } \\ s & \text { Fin spacing, mm } \\ t & \text { Fin thickness, mm } \\ \mathrm{u} & \text { Velocity component in } \mathrm{x} \text { direction }\end{array}$

\section{Greek Symbols}

\begin{tabular}{ll}
$\mathrm{E}$ & Turbulence dissipation rate, $\mathrm{m}^{2} / \mathrm{s}^{3}$ \\
$\mathrm{k}$ & Turbulent kinetic energy, $\mathrm{m}^{2} / \mathrm{s}^{2}$ \\
$\rho$ & Density of the air, $\mathrm{kg} / \mathrm{m}^{3}$ \\
$\varphi$ & Generalized transport variable \\
$\mathrm{I}$ & Effective diffusivity, $\mathrm{m}^{2} / \mathrm{s}^{2}$ \\
\hline
\end{tabular}

\footnotetext{
*Corresponding author: K V Ramana Murthy, scientist ' $D$ ', research fields: compact plate fin heat exchangers, condensation and heat transfer. E-mail: katabathuni@jetmail.ada.gov.in.
}

\section{Introduction}

Plate-fin compact heat exchangers are widely used in aerospace, automobile and cryogenic industries due to its compactness (i.e., high heat transfer surface area-to-volume ratio) for desired thermal performance, resulting in reduced space, weight, support structure, footprint, energy requirement and cost. Depending on the application, various types of augmented heat transfer surfaces such as wavy fins, Serrated fins, Louvered fins and Perforated fins are used in compact heat exchangers. The high rate of heat exchange is made possible by employing intricate flow passages with large heat transfer areas. It is important to know the heat transfer and flow friction characteristics ( $j$ and $f$ factors) of the enhanced surfaces for a proper selection and rating of the equipment. Serrated fins have a high degree of surface compactness and substantial heat transfer enhancement obtained as a 
result of the periodic starting and development of laminar boundary layers over interrupted channels formed by the fins and their dissipation in the fin wakes. There is, of course, an associated increase in the pressure drop due to increased friction and form-drag contribution from the finite thickness of the interrupted fins. Thermo-hydraulic design of a compact heat exchanger is strongly dependent upon the performance of heat transfer surfaces. An extensive literature survey has been carried out to find a correct correlation on the water side heat transfer coefficient for serrated fins. The colburn and friction factor correlations presented by Pallavi and Ranganayakulu [1], using CFD are compared with Wieting [2], Kays and London [3], Mochizuki et al. [4], Manglik and Bergles [5] and Joshi and Webb [6], which were obtained from the experiments with air flow. Also, the colburn and friction factor correlations presented by Rao et al. [7], using CFD are compared with Kays and London [3], which were obtained from the experiments with air flow for the plain fins. Sen Huand Herold [8, 9] have published the affects of the Prandtl number on Colburn $j$ factor for water and PAO (Polyalphaolefin) fluids and claimed that, air models over-predict the $j$ factor for liquids and found that the heat transfer coefficient of liquids is approximately two times larger than that of air. The flow for a larger Prandtl number has a longer thermal developing region on the channel wall and on each fin, which leads to a higher average heat transfer rate but a smaller Colburn factor. Kim and Sohn [10] proposed that the Colburn factors of the liquid were about $25 \%$ smaller than air. Hence, a detailed analysis had been carried out using ANSYS Fluent tool to estimate the $j$ and $f$ factors for serrated fins. The correlations also allow the effect of fin length, height, thickness, spacing and hydraulic diameter on the performance to be assessed.

Among the very few numerical analysis for generation of heat transfer and pressure drop characteristics of Serrated fin heat exchanger was studied by Bhowmik and Kwan [11]. They recalculated the Nusselt number using the correlated $j$ and the effects of Prandtl numbers were studied. They also indicated that an air model should not be used to predict the fluid flow and heat transfer of Serrated fin heat exchangers operated with liquid media.

\section{Mathematical Model}

The assumptions made in the CFD simulation are the flow is stable in the computational domain and the fluid in the domain is incompressible. In this work, the CFD package ANSYS Fluent 14.5 is used for simulation. In ANSYS Fluent, the conservation equations of mass, momentum and energy are solved using the finite volume method. The turbulent flow is calculated by the semi-implicit SIMPLER as mentioned in Versteeg and Malalasekera [12].

A standard $k-\varepsilon$ model as given in Versteeg and Malalasekera [12] with enhanced wall treatment is used to predict turbulent flow in the fin geometry. The Reynolds transport equations can be written in a generalized form as given by Anderson [13] and Patankar [14].

$$
\operatorname{div}(\rho u \varphi)=\operatorname{div}(I \operatorname{grad} \varphi)+S_{\varphi}
$$

where, $\varphi$ stands for a generalized transport variable, which is used for all conserved variables in a fluid flow problem, including, mass, momentum and the turbulence variables $k$ and $\varepsilon . \digamma$ represents the effective diffusivity (sum of the eddy diffusivity and the molecular diffusivity). $S_{\varphi}$ is the source term for the respective dependent variable. The solution of the above set of equations is applied to the prediction of velocity and turbulence levels throughout the domain. The convergent criterion is specified to absolute residuals $\left(\leq 1.0 \times 10^{-6}\right)$.

\section{Problem Description}

The geometrical features of the three dimensional Serrated fin are described by the fin height $(h)$, fin spacing $(s)$, fin thickness $(t)$ and offset strip length $(l)$. The dimensionless representations of these variables are given by the flow cross-section aspect ratio $(\mathrm{s} / \mathrm{h}), \mathrm{t} / \mathrm{s}$ 
and $\mathrm{t} / \mathrm{l}$. These dimensional notations are shown in Fig 1 .

The performance of a plate fin surface is not uniquely determined by the hydraulic diameter. Other geometric parameters such as the fin height $(h)$, fin spacing $(s)$, fin thickness $(t)$ and offset strip length $(l)$ play significant role as indicated by London and Shah [15]. It will be prohibitively expensive and time consuming to fabricate heat exchanger cores and conduct experiments over reasonable ranges of all the geometric variables. In contrast, it is relatively easy and cost effective to carry out parametric study through numerical simulation and derive acceptable correlations for use in industry.

Several authors have contributed significantly in some or the other way to develop a correlation that can take into account all the possible effects in order to determine $j$ and $f$, but none of them had the same conclusions. There was no unique correlation developed by any one that can be relied upon in order to determine $j$ and $f$ especially for the offset strip fins using water. So, this work mainly focused to develop good correlations for serrated fins taking all the necessary parametric effects using water medium.

The optimum Serrated fin surfaces are taken for parametric study. A total of 14 different fin surfaces are modeled and CFD analysis is carried out for developing correlations. Numerical procedure has been used to find out $j$ and $f$ factors for the serrated fin surfaces with in the ranges of dimensionless parameters of offset fin surfaces. The range of dimensionless geometric parameters and Reynolds number are taken as follows:

$$
\begin{aligned}
& 0.186 \leq s / h \leq 0.568 \\
& 0.0765 \leq t / s \leq 0.1675 \\
& 0.027 \leq t / l \leq 0.082 \\
& 100 \leq R e \leq 1,000 \text { Laminar region } \\
& 1,000 \leq R e \leq 15,000 \text { Turbulent region }
\end{aligned}
$$

The performance factors are evaluated for different types of serrated fins using ANSYS Fluent. The performance of serrated fins are presented for both laminar and turbulent regions of Re vs. $j$ and $f$ factors.

\section{CFD Approach}

The CFD analysis is carried out using ANSYS Fluent for an estimation of $j$ and $f$ factors for serrated fin geometry for water medium. A number of numerical experiments carried out to determine the thermo-hydraulic parameters with a view to arrive at the optimum parameters for a serrated fin. The three dimensional computational domain of serrated fin model shown in Fig. 2 is analyzed using CFD simulation tool ANSYS Fluent. The CFD analysis is carried out for different geometric parameters and Reynolds numbers using ANSYS Fluent 14.5 in HP Z800 workstation with 20 GB RAM. The analysis is carried out in two phases. In first phase, serrated fin is taken and characterized for $f$ values over a range of Reynolds number. In second phase, the $j$ value is determined for the same range by switching on the energy equation. The mass flow rates are determined for

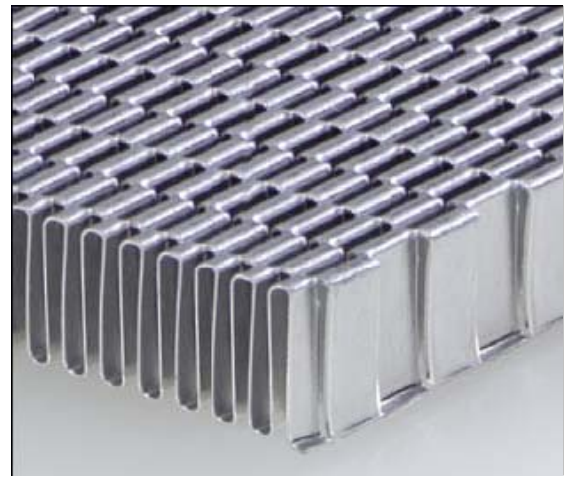

(a)

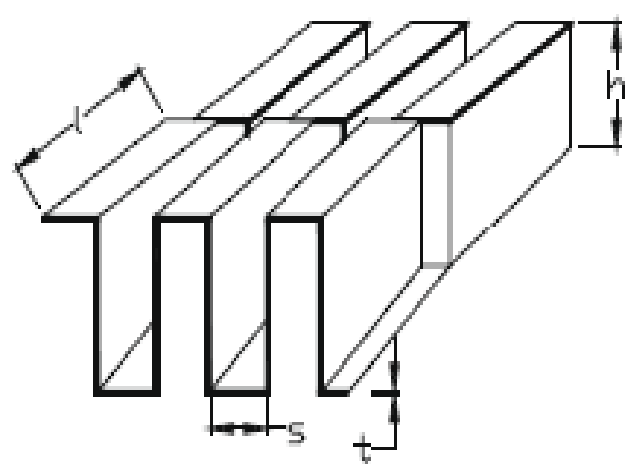

(b)

Fig. 1 Schematics of fin geometry: (a) Serrated fin - isometric view and (b) serrated fin dimensional notations. 
a range of Reynolds number from 100 to 15,000 . In order to overcome the entrance effect, the concept of periodic fully developed flow as suggested by Patankar et al. [16] is implemented for flow analysis. After the analysis, the pressure drop for unit length is one of the outputs, and that is multiplied by the actual length to get the total pressure drop for corresponding fins. From the pressure drop, friction factor is calculated as per Appendix A of Kays and London [3]. Finally, the corresponding two-dimensional fully developed velocity profile is listed out. Similarly, the same procedure is repeated for the range of Reynolds numbers from 100 to 15,000 in order to draw the $f$ vs. $R e$ curves. The mesh finalization is done before analyzing for the $j$ and $f$ factors. The grid independency curve is drawn between number of elements and the pressure drop parameters. Then the mesh size is determined based upon the curve where the slope is almost zero as shown in Fig. 3.

In second phase, the "velocity inlet" and "outflow" boundary conditions are used at the inlet and outlet of the fin geometry respectively. The two-dimensional fully developed velocity profile, which is taken from first phase analysis (pressure drop analysis), is used in the "velocity inlet" boundary condition.

The assumption of constant wall temperature boundary condition is employed for walls. After the thermal analysis, post processing is done for temperatures and pressures at the inlet and outlet using mass weighted average, and pressure, temperature and velocity profiles are taken at the various sections of the fins for corresponding Reynolds numbers. This temperature difference between inlet and outlet of the fin, in turn, is used for calculating $j$ factor using Kays and London [3] methodology. Similarly, the same procedure is repeated for the range of Reynolds numbers 1,000 to 15,000 for turbulent flow and from 100 to 1,000 for laminar flow in order to draw the $j$ vs. $R e$ characteristic curves. The actual mass flow rate is used as the boundary condition. No-slip boundary condition is used for walls. The $j$ and $f$ data are generated as stated above.

\section{Validation}

Serrated fin has been analyzed with CFD and compared with experimental data. The results are further compared with open literature. Analysis has been carried out by considering uniform wall temperature boundary condition. For validation of the numerical analysis conducted in the present study, a grid independence test is carried out for the same fin and graph is plotted as the number of elements verses pressure drop as shown in Fig. 3. It shows that after 280,000 cells there is not much variation in the pressure drop.

The results obtained from ANSYS Fluent in the form of Colburn $j$ and Fanning friction factors are compared with the literature correlations for air and water medium

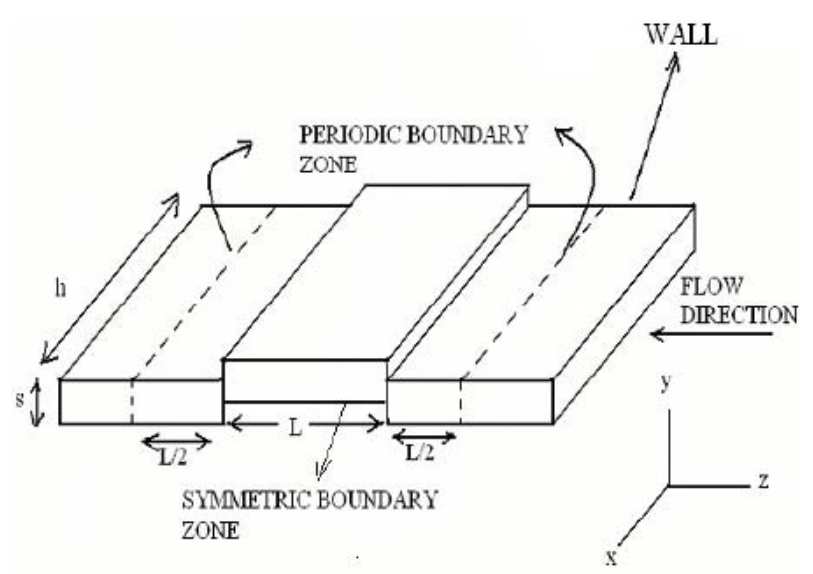

Fig. 2 Computational domain for serrated fin.

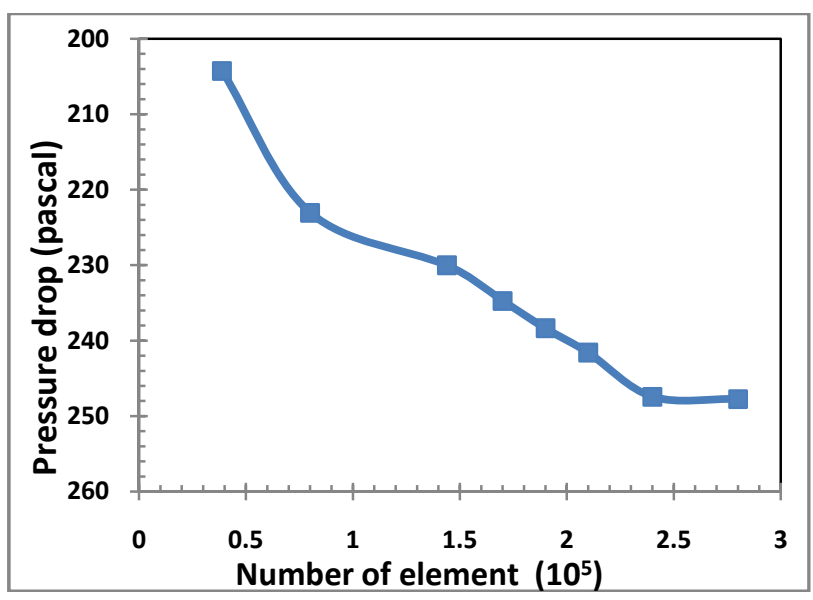

Fig. 3 Grid independency graph - CFD analysis. 
in Fig. 4 for laminar region and turbulent region respectively. According to Sen Huand Herold [8, 9], there will be a considerably large deviation in $j$ values of air with water due to a large difference in Prandtl number and same was observed. The CFD results of serrated fins are compared with Wieting [2], Sen Huand Herold [8, 9] and Kim and Sohn [10] as shown in Fig. 4. The CFD results are in good agreement with the analytical results given by Sen Huand Herold [5, 6] for the low Reynolds number region. The variations are found to be $5-14 \%$ in $j$ values. Also, Fig. 4 shows the comparison of FLUENT results with the literature correlations [8-10]. It is evident for $f$ and $j$ factors that Sen Huand Herold [8, 9] correlations are close to FLUENT results. However some variations are observed due to the fin geometries when compared with FLUENT data. Giving exact reasons for variations of these factors may not be possible due to involvement of so many parameters such as manufacturing aspects testing conditions, etc.

It is found that the $j$ of water is lower by about two times when compared with air, where as there is no significant deviation in $f$ values as observed by Sen Huand Herold $[8,9]$. Even though $j$ is lower for water, the heat transfer coefficient, $\mathrm{h}_{\mathrm{w}}$ is much higher when compared to air. The $f$ vs. $R e$ and $j$ vs. Re data for serrated fin surface shows significant nonlinearity over the Reynolds number range $100 \leq R e \leq 15,000$. Therefore, two separate equations have been proposed for the low and the high Re regimes.

\section{Results and discussion}

The CFD analysis for serrated fin surfaces is carried out using ANSYS Fluent with boundary conditions and respective mass flow rates for various Reynolds numbers. From the Fig. 4 , it is observed that the $j$ and $f$ vs. Re curves of serrated fins follow the same trends as Sen Huand Herold [8, 9] and Kim and Sohn [10] experimental results. The effects of variation of dimensionless geometrical parameters on Serrated fin performance are presented in the subsequent para. The response of velocity, pressure and temperature fields to changes in geometric parameters and Reynolds number is clearly manifested. The Figs. 5-10 illustrate the role of geometric parameters $s / h, t / s, t / l$ vs. $j$ and parameters $s / h, t / s, t / l$ vs. $f$ in determining the heat transfer and flow friction performance of a Serrated fin surfaces. The individual effects of geometrical parameters are explained in the following paragraphs:

\subsection{Effect of $s / h$ Ratio on $j$}

The Colburn $j$ factors are plotted against the fin

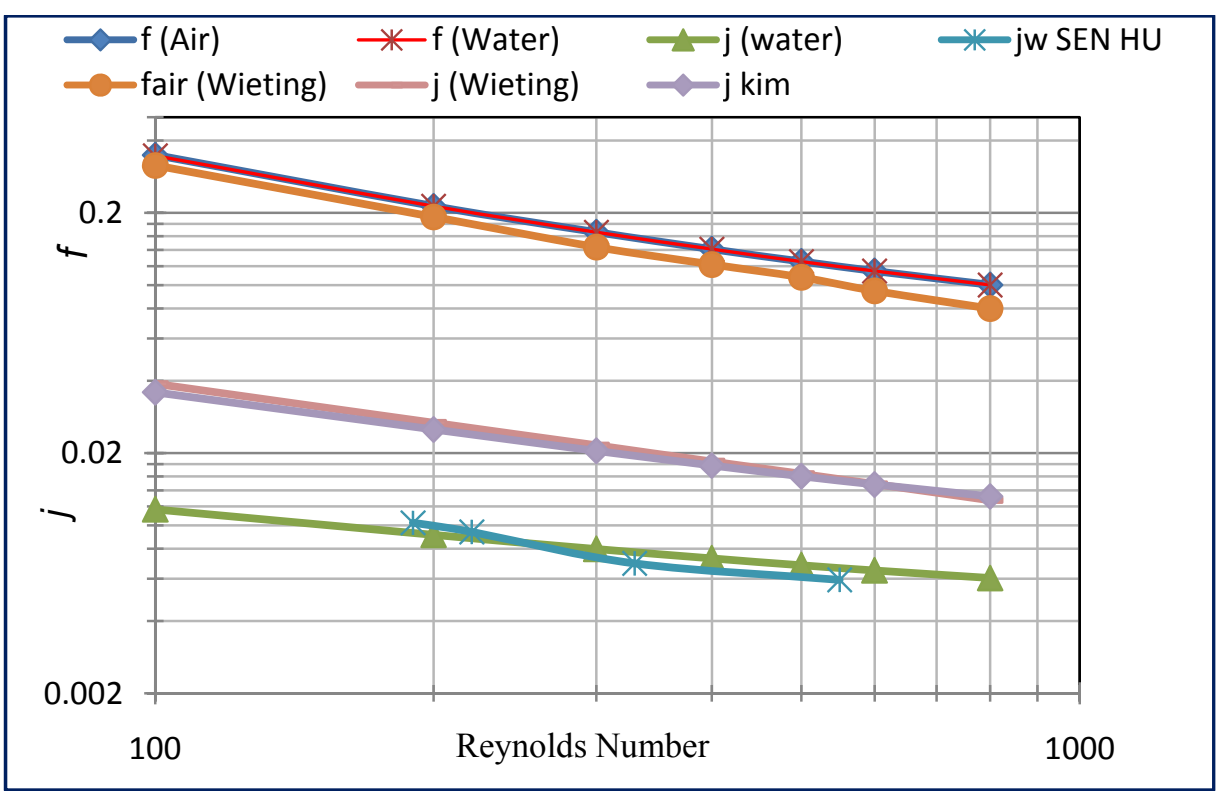

Fig. 4 Validation of CFD results with experimental results. 
spacing $(s)$ to fin height $(h)$ ratio $(0.186$ to 0.568$)$ for various Reynolds numbers as shown in Fig. 5. The $j$ factor decreases with $R e$ number increases as expected. The rate of decrease is higher for low Reynolds numbers and lower for high Reynolds number. However, the ratio of increase is predominant for low $R e$ (below 1,000) and it is not significant for high $R e$ (above 1,000). The increase of this factor is mainly due to higher value of hydraulic diameter. As aspect ratio increases, the hydraulic diameter is also increased.

\subsection{Effect of $t / s$ Ratio on $j$}

The Colburn $j$ factors are plotted against the fin thickness $(t)$ to fin spacing $(s)$ ratio $(0.0765$ to 0.1675$)$ for various Reynolds numbers as shown in Fig. 6. It is observed that as Reynolds number increases, $j$ factors are coming down due to same reasons as mentioned above. Also, it is noted that the $j$ factor decrease with increase of $t / s$ ratio (up to 0.15 ) for all Reynolds numbers. As $t / s$ ratio increases, the recirculation zone tends to diminish due to higher flow velocity in the offset passages. This leads to decrease in heat transfer rate.

\subsection{Effect of $t / l$ Ratio on $j$}

The Colburn $j$ factors are plotted against the

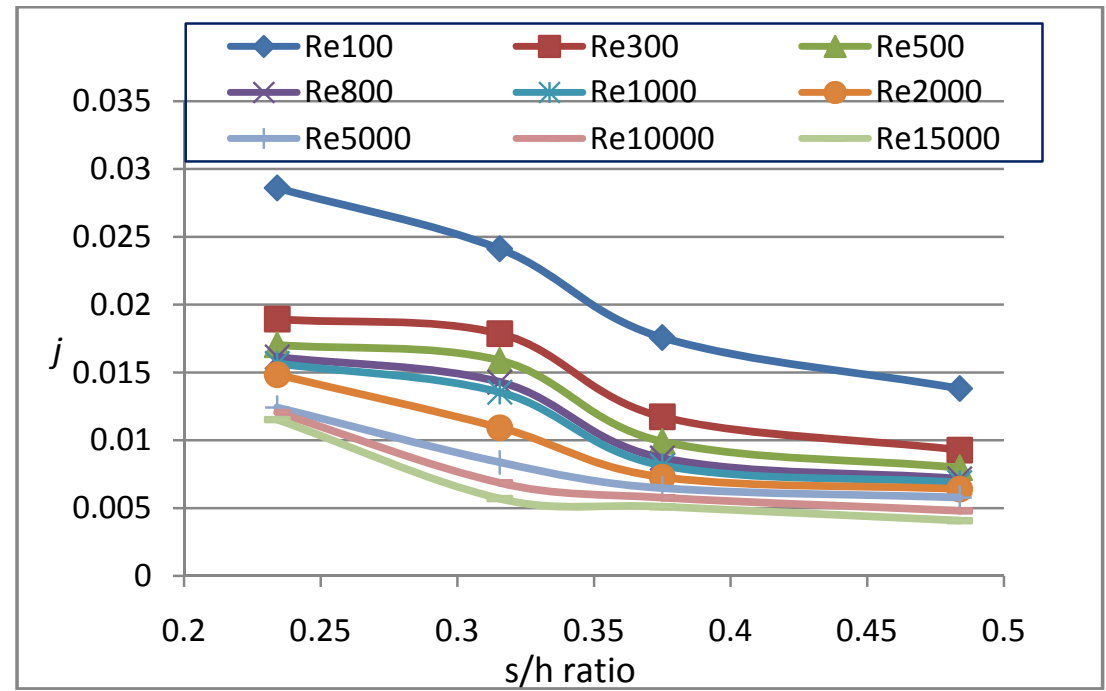

Fig. 5 Dimensionless Parameter $s / h$ ratio Vs. $f$ for different $R e$.

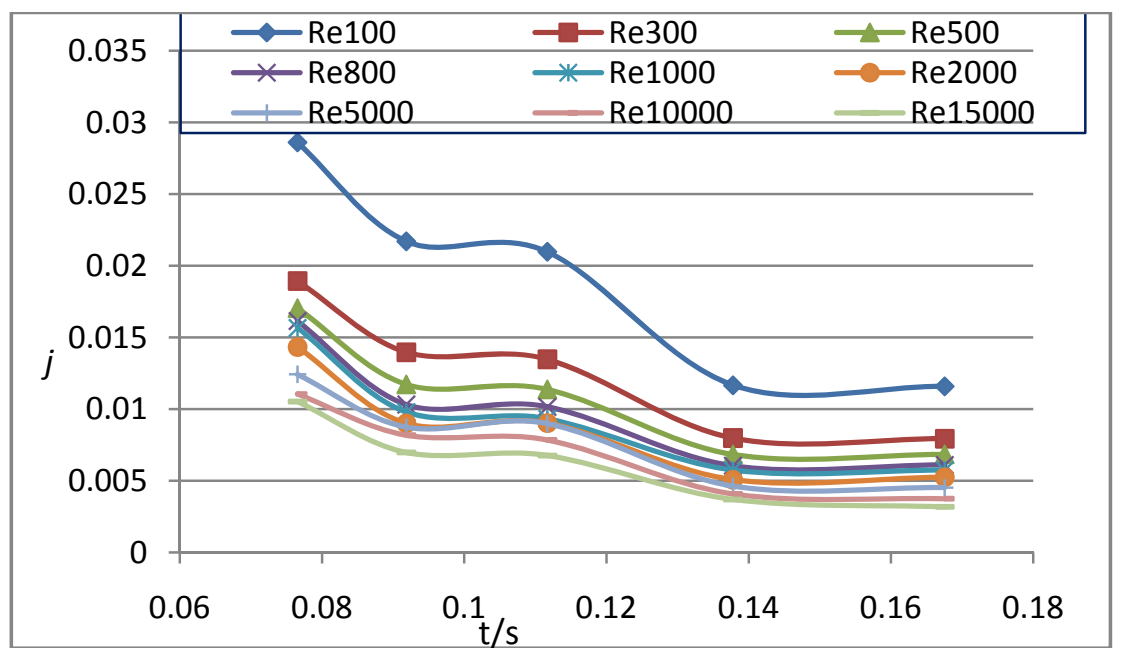

Fig. 6 Dimensionless parameter $t / s$ ratio vs. $j$ for different $R e$. 
fin-thickness $(t)$ to fin length $(l)$ ratio $(0.027$ to 0.082$)$ for various Reynolds numbers as shown in Fig. 7. It is observed that as Reynolds number increases, $j$ factors are coming down due to same reasons as mentioned above.The value of $j$ decreases with increase of $t / l$ for all Reynolds numbers. Also, it is noted that the $j$ factor decreases with increase of $t / l$ ratio (up to 0.05 ) for all Reynolds numbers. It is found that $t / l$ ratio increases the recirculation zone at the interrupted layer increases.

\subsection{Effect of s/h Ratio on $f$}

The friction factor $f$ is plotted against the fin spacing $(s)$ to fin height $(h)$ ratio as shown in Fig. 8. The $f$ factor decreases with $R e$ number increases as expected. As aspect ratio increases, the hydraulic diameter is also increased.

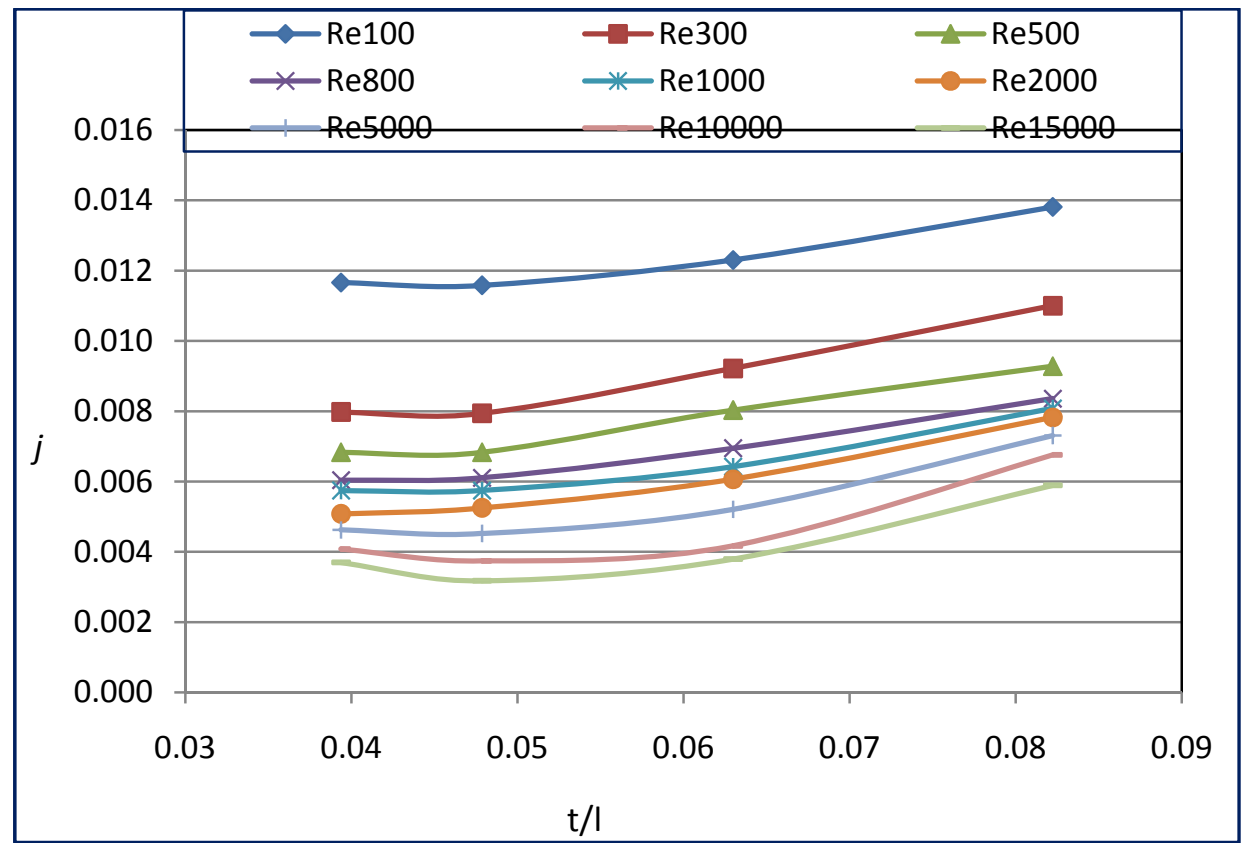

Fig. 7 Dimensionless parameter $t / l$ ratio vs. $j$ for different $R e$.

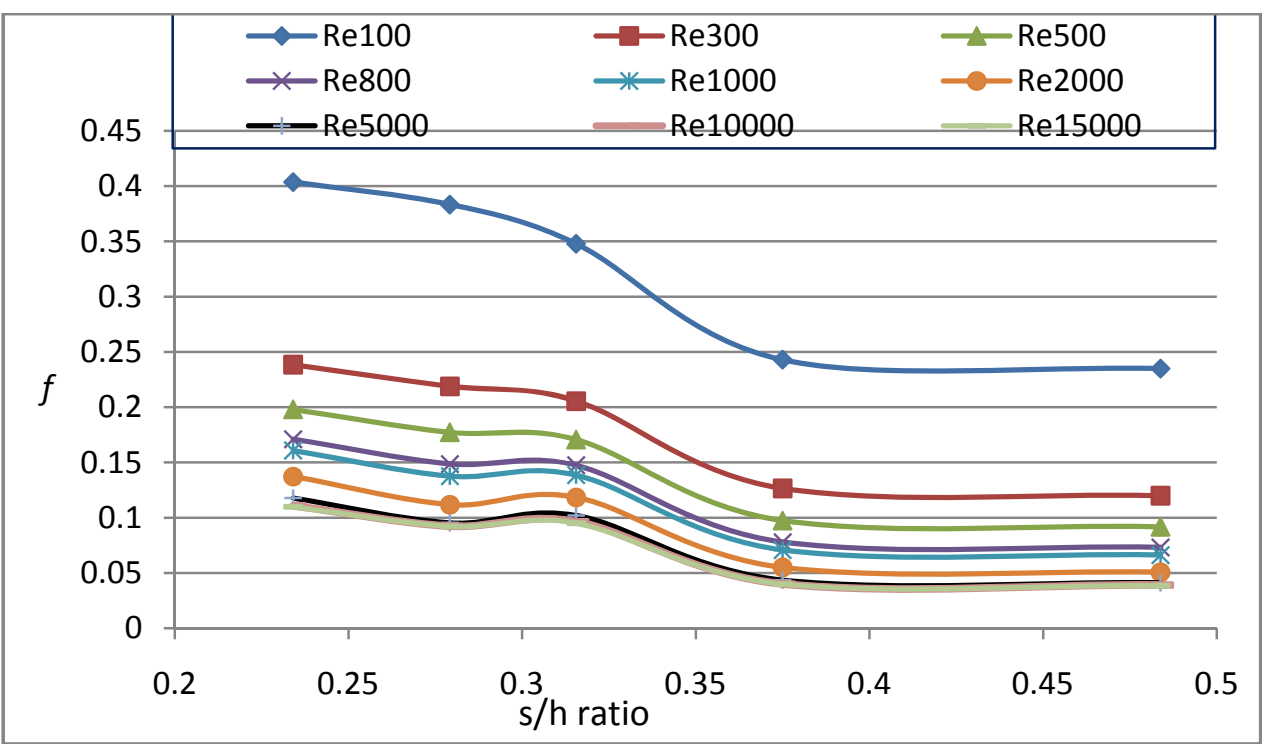

Fig. 8 Dimensionless parameters/h ratio vs. $f$ for different $R e$. 


\subsection{Effect of t/s Ratio on $f$}

The friction factor $f$ is plotted against the fin thickness $(t)$ to fin spacing $(s)$ ratio $(0.0765$ to 0.1675$)$ for various Reynolds numbers as shown in Fig. 9. It is observed that as Reynolds number increases, $f$ factors are coming down due to same reasons as mentioned above.

\subsection{Effect of t/l Ratio onf}

The friction factor $f$ is plotted against the fin-thickness $(t)$ to fin length $(l)$ ratio $(0.027$ to 0.082$)$ for various Reynolds numbers as shown in Fig. 10. It is observed that as Reynolds number increases $f$ factors are coming down.

\subsection{Velocity, Temperature and Pressure Contours}

The velocity vector for fin surface $5 \mathrm{~h}-28 \mathrm{FPI}-0.127 \mathrm{t}$, the Reynolds numbers 500 and 10,000 are shown in Fig. 11 and 12 for comparison of velocity magnitudes. From the velocity vectors it is quite clear that the flow

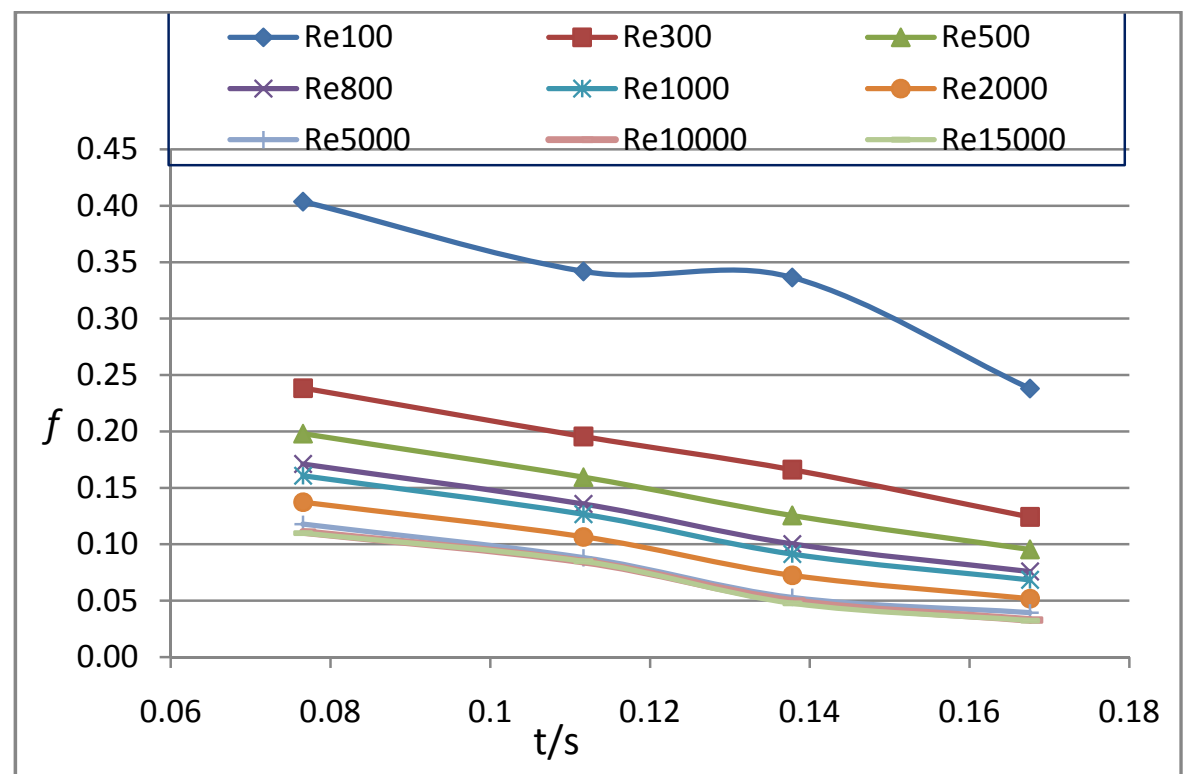

Fig. 9 Dimensionless parameter $t / s$ ratio vs. $f$ for different $R e$.

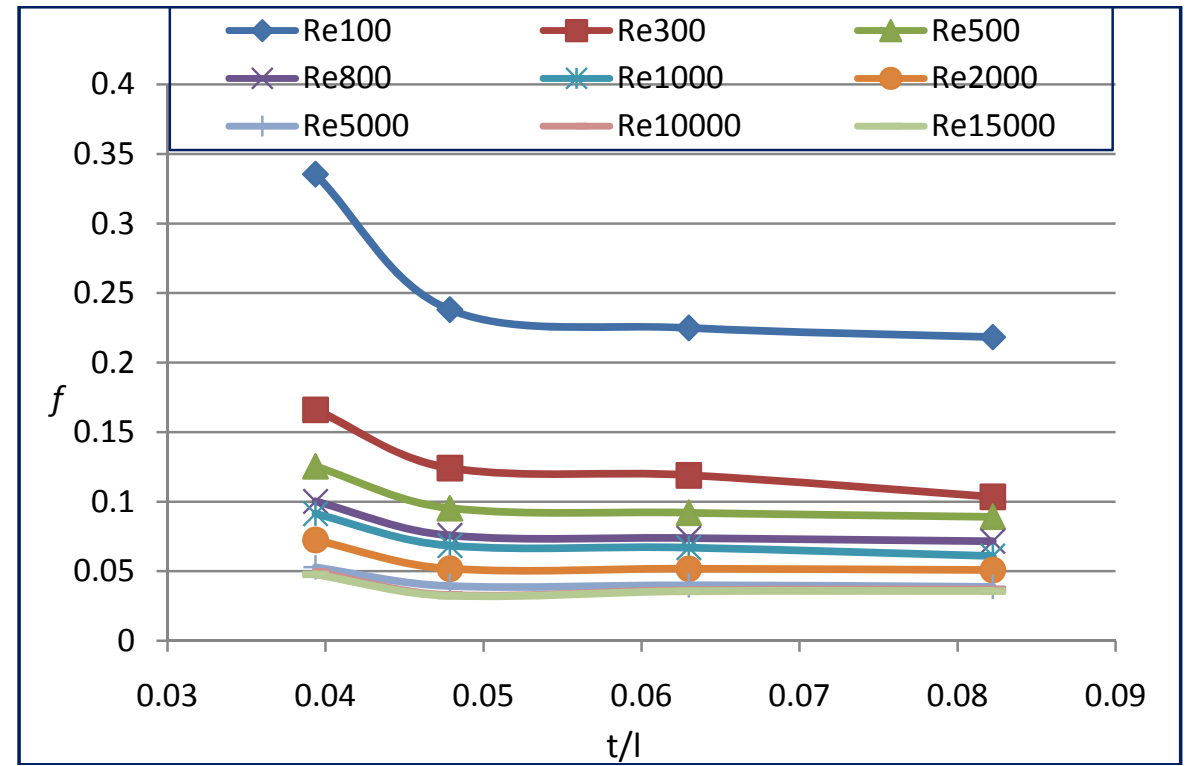

Fig. 10 Dimensionless parameter $t / l$ ratio vs. $f$ for different $R e$. 


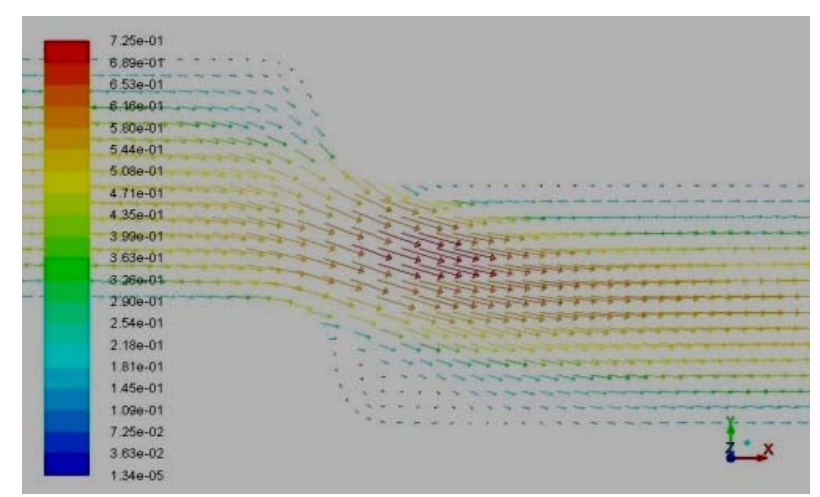

Fig. 11 Velocity vector at $R e=500$.

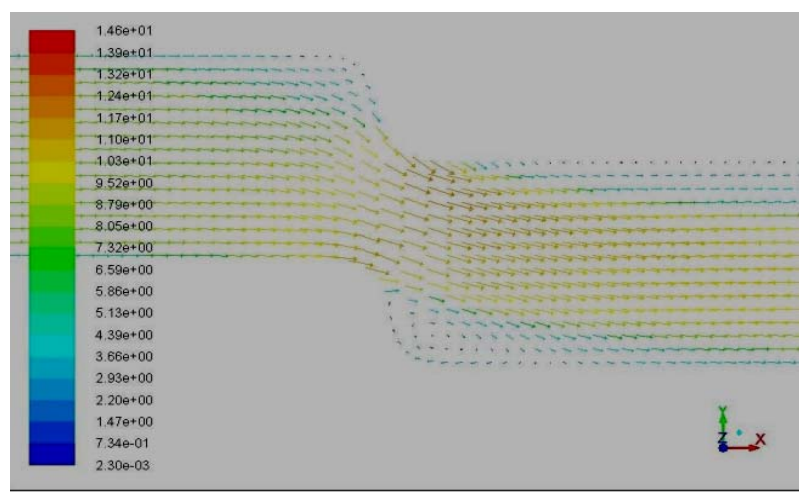

Fig. 12 Velocity vector at $R e=10,000$.

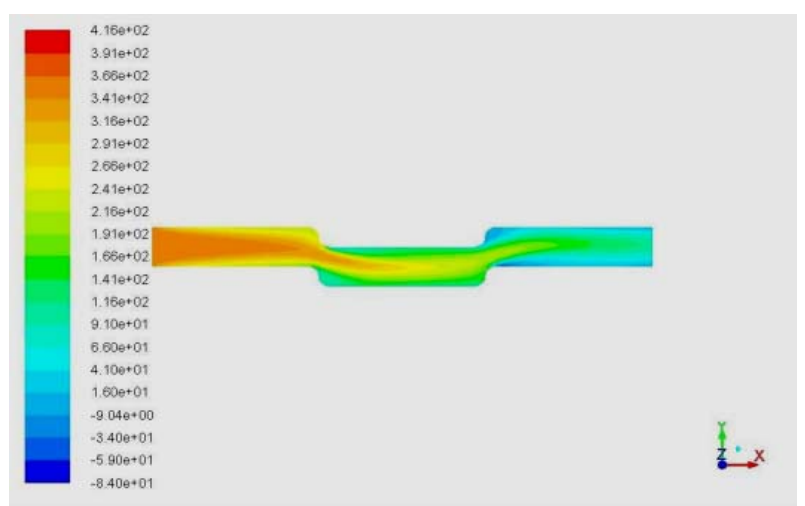

Fig. 13 Pressure contour at $R e=500$.

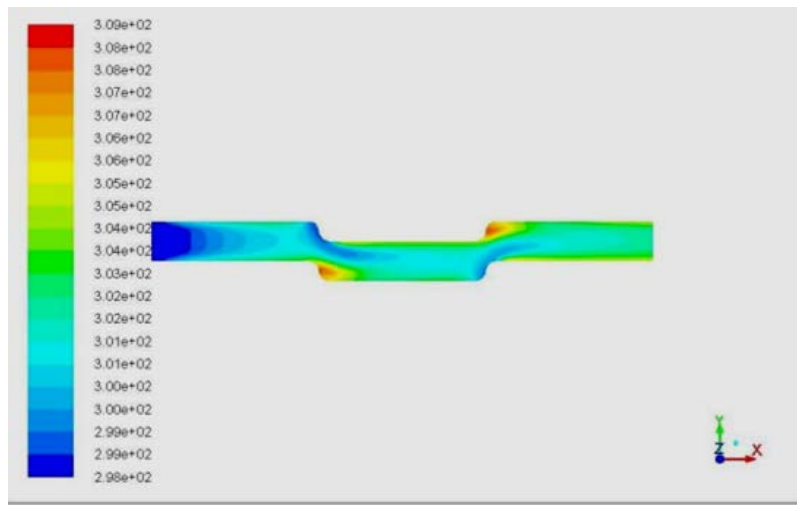

Fig. 14 Temperature contour at $R e=500$. is more laminar at Reynolds number 500 and is turbulent for the Reynolds number 10,000. This is because the velocity profile is more parabolic in shape for the Reynolds number 500 and is more flat for the Reynolds number 10,000 . One more interesting feature is the shape of the vectors, which are pulled towards the interrupted part. This is basically because of the formation of the recirculation zone that is pulling the velocity contours towards it in order to compensate for the excess pressure drop due to the recirculation pocket.

The pressure and temperature contours are shown in Fig. 13 and 14 respectively for the same fin surface. It is clear that the boundary layer interrupts and fresh boundary layer starts from the interruption. This is more predominantly seen for the low Reynolds number case (Re 500). Moreover, high pressure drop occurs at the interruptions and the velocity reaches its maximum value at the same place.

\section{Correlations for $\boldsymbol{j}$ and $\boldsymbol{f}$ Factors}

An exhaustive numerical study has been carried out on the heat transfer phenomena in plate fin surfaces with serrated fins. The $j$ vs. Re and $f v s$. Re curves shows significant non-linearity as shown in the above Figs. The correlations have been expressed in terms of two separate equations over the low and high $R e$ regions along with dimensionless geometric parameters. The power law expressions have been used for determining the Colburn factor $j$ and friction factor $f$ as a function of the Reynolds number and dimensionless fin parameters.

The colburn factor $j$ are functionally related to $R e$, $\mathrm{s} / \mathrm{h}, \mathrm{t} / \mathrm{s}$ and $\mathrm{t} / \mathrm{l}$, and it can be represented as

$$
j=\mathrm{B} \cdot \operatorname{Re}^{\mathrm{a} 1}(\mathrm{~s} / \mathrm{h})^{\mathrm{a} 2}(t / s)^{\mathrm{a} 3}(t / l)^{\mathrm{a} 4}
$$

where, B, a1, a2, a3 and a4 are constants.

The use of these power law expressions is justified because variations in $j$ with $R e, s / h, t / s$ and $t / l$ follow constant slope log-linear lines in both laminar and fully turbulent flow regions. The $j$ vs. Re data for Serrated fin surfaces shows significant nonlinearity over the 
Reynolds number range $100 \leq R e \leq 15,000$. Therefore, two separate equations have been proposed for the low and the high Re regions as follows:

$$
\begin{aligned}
& j=0.426 \operatorname{Re}^{-0.308}(s / h)^{0.585}(t / s)^{-0.929}(t / l)^{0.943} \\
& \text { for Laminar range }(100 \leq R e \leq 1,000) \\
& j=0.097 \operatorname{Re}^{-0.151}(s / h)^{0.526}(t / s)^{-1.238}(t / l)^{1.033}
\end{aligned}
$$

for Turbulent range $(1,000 \leq R e \leq 15,000)$

The above correlations correctly predict $94 \%$ of the $j$ data for laminar regime, and $93 \%$ of the $j$ data for the turbulent regions lies within $\pm 10 \%$ and root-mean-square error is $2.8 \%$.

Similarly, the fanning friction factor $f$ are functionally related to $R e, s / h, t / \mathrm{s}$ and $t / l$, and it can be represented as

$$
f=\mathrm{C} \cdot \operatorname{Re}^{\mathrm{b} 1}(s / h)^{\mathrm{b} 2}(t / s)^{\mathrm{b} 3}(t / l)^{\mathrm{b} 4}
$$

where, $\mathrm{C}, \mathrm{b} 1, \mathrm{~b} 2, \mathrm{~b} 3$ and $\mathrm{b} 4$ are constants.

The use of these power law expressions is justified because variations in $f$ with $R e, s / h, t / s$ and $t / l$ follow constant slope log-linear lines in both laminar and fully turbulent flow regions. The $f$ vs. Re data for Offset fin surfaces show significant nonlinearity over the Reynolds number range $100 \leq R e \leq 15,000$. Therefore, two separate equations have been proposed for the low and the high $R e$ regions as follows:

$$
\begin{aligned}
& f=3.152(\mathrm{Re})^{-0.481}(\mathrm{~s} / \mathrm{h})^{-0.272}(t / s)^{1.237}(t / l)^{0.984} \\
& \text { for Laminar range }(100 \leq \operatorname{Re} \leq 1,000) \\
& f=0.421(\mathrm{Re})^{-0.205}(\mathrm{~s} / \mathrm{h})^{-0.135}(t / s)^{-1.673}(t / l)^{1.194} \\
& \text { for Turbulent Range }(1,000 \leq \operatorname{Re} \leq 15,000)
\end{aligned}
$$

The above correlations correctly predict $92 \%$ of the $j$ data for laminar regime and turbulent regions lies within $\pm 12 \%$ and root-mean-square error is $6.7 \%$.

\section{Conclusions}

This paper presents the heat transfer correlations and the pressure drop for serrated fins in water media, which are widely used in Aerospace industry. The expressions provided for the heat transfer coefficient in terms of Colburn $j$ factor and frication factor $f$ allows the computation for all values of Reynolds number, including the laminar and turbulent regions. The generalized correlations are developed for Serrated fins taking all geometrical parameters into account for entire range of operations of Aerospace industry. In addition, the effects of various geometrical parameters are presented as parametric studies. These expressions are well formed in the laminar and fully turbulent regions, since they can be considered as the standard expressions modified by correction factors. The values obtained from these expressions are in agreement with the literature data. The application of heat transfer and friction factor expressions to a compact plate type heat exchanger gives very good agreement within 15\% experimental data. The above correlations can be used by heat exchanger designers and can reduce the number of tests and modification of the prototype to a minimum for similar applications.

\section{Acknowledgements}

The Authors wish to acknowledge Aeronautical Development Agency for allowing publication of the paper.

\section{References}

[1] Pallavi, P. and Ranganayakulu, C. 2011. "Development of Heat Transfer Coefficient and Friction Factor Correlations for Offset Fins Using CFD." Journal of Numerical Methods for heat and fluid flow 21: 935-51.

[2] Wieting, A. R. 1975. "Empirical Correlations for Heat Transfer and Flow Friction Characteristics of Rectangular Offset-Fin Plate-Fin Heat Exchangers." Journal of Heat Transfer 97: 488-90.

[3] Kays, W. M. London, A. L. 1964. Compact Heat Exchangers. Reprint Third edition, Krieger Publishing.

[4] Mochizuki, S., Yagi, Y. and Yang, W. J. 1987. "Transport Phenomena in Stacks of Interrupted Parallel-Plate Surfaces." Experimental Heat Transfer 1: 127-40.

[5] Manglik, R. M., Bergles, A. E. 1995. "Heat Transfer and Pressure Drop Correlations for the Rectangular Offset Fin Compact Heat Exchanger." J Experimental Thermal and Fluid Science 10: 171-80.

[6] Joshi, H. M. and Webb, R. L. 1987. "Heat Transfer and Friction in the Offset Strip - Fin Heat Exchanger." International Journal of Heat and Mass Transfer 30: 60-84.

[7] Rao, R. B. S., Ranganath, G. and Ranganaykulu, C. 2013. "Development of Colburn ' $j$ ' Factor and Fanning Friction Factor ' $f$ ' Correlations for Compact Heat Exchangers Plains fins by Using CFD." International Journal of Heat 
and Mass Transfer 49: 991-1000.

[8] Sen, H. S. and Herold, K. E. 1995. "Prandtl Number Effect on Offset Fin Heat Exchanger Performance: Predictive Model for Heat Transfer and Pressure Drop." Int J Heat Mass Transfer 38: 1043-51.

[9] Sen, H. S. and Herold, K. E. 1995. "Prandtl Number Effect on Offset Fin Heat Exchanger Performance: Experimental Results." Int J Heat Mass Transfer 38: 1053-61.

[10] Kim, B. and Sohn, B. 2006. "An Experimental Study of Flow Boiling in a Rectangular Channel with Offset Strip Fins." International Journal of Heat and Fluid Flow, 27: 514-21.

[11] Bhowmik, H. and Kwan, S. L. 2009. "Analysis of Heat Transfer and Pressure Drop Characteristics in an Offset Strip Fin Heat Exchanger.” International Communications in Heat and Mass Transfer 36:259-63.

\section{Research Limitations}

The present numerical analysis is carried out for "water" media, and hence these results may not be accurate for other fluids with large variations of Prandtl numbers.

\section{Practical Implications}

In open literature, these fins are generally evaluated as a function of Reynolds number experimentally, which are expensive. However, their performance will also depend to some extent on geometrical parameters such as fin thickness, fin spacing, fin lance

length and fin height.
[12] Versteeg, H. K. and Malalasekera, W. 1995. An Introduction to Computational Fluid Dynamics - The Finite Volume Method. Prentice Hall Publications, England.

[13] Anderson, J. D. 1995. Computational Fluid Dynamics-The Basic with Applications. Mc Graw-Hill Companies, Inc, New York.

[14] Patankar, S.V. 1980. "Numerical Heat Transfer and Fluid Flow." Hemisphere Publications.

[15] London, A. L. and Shah, R. K. 1968. "Offset Rectangular Plate Fin Surfaces - Heat Transfer and Flow Friction Characteristics." ASME. Eng. Power 90 (A): 218-28.

[16] Patankar, S. V., Liu, C. H. and Sparrow, E. 1977. "Fully Developed Flow and Heat Transfer in Ducts Having Stream wise-Periodic Variations of Cross-Sectional Area." Journal of Heat Transfer 99: 180-6. 